\title{
Offshore Wind Measurements using Doppler Aerosol Wind Lidar (DAWN) at NASA Langley Research Center
}

\author{
Jeffrey Y. Beyon ${ }^{1}$, Grady J. Koch'², and Michael J. Kavaya ${ }^{3}$ \\ ${ }^{1}$ NASA Langley Research Center, MS 488, Hampton, VA 23681, USA, Jeffrey.Y.Beyon@nasa.gov \\ ${ }^{2}$ NASA Langley Research Center, MS 468, Hampton, VA 23681, USA, Grady.J.Koch@nasa.gov \\ ${ }^{3}$ NASA Langley Research Center, MS 468, Hampton, VA 23681, USA, Michael.J.Kavaya@nasa.gov
}

\begin{abstract}
The latest flight demonstration of Doppler Aerosol Wind Lidar (DAWN) at NASA Langley Research Center (LaRC) is presented. The goal of the campaign was to demonstrate the improvement of DAWN system since the previous flight campaign in 2012 and the capabilities of DAWN and the latest airborne wind profiling algorithm APOLO (Airborne Wind Profiling Algorithm for Doppler Wind Lidar) developed at LaRC. The comparisons of APOLO and another algorithm are discussed utilizing two and five line-of-sights (LOSs), respectively. Wind parameters from DAWN were compared with ground-based radar measurements for validation purposes. The campaign period was June - July in 2013 and the flight altitude was $8 \mathrm{~km}$ in inland toward Charlotte, NC, and offshores in Virginia Beach, VA and Ocean City, MD. The DAWN system was integrated into a UC12B with two operators onboard during the campaign.
\end{abstract}

Keywords: Wind profile algorithm, coherent lidar, APOLO, DAWN AIR

\section{INTRODUCTION}

The latest flight campaign of DAWN instrument in 2013 is presented in this paper. The Data Acquisition, Processing, and Display System (DAPDS) of the DAWN system is in charge of real-time data acquisition and processing [6,7]. DAPDS also controls the scanner to steer the lidar beam to desired LOSs and the Inertial Navigation System (INS) Global Positioning System (GPS) unit to acquire the motion and orientation information of the instrument and the aircraft. The focus of this paper is on the wind products estimated from the data during such campaign [1]. A brief description of the airborne data processing algorithm APOLO is presented as well [4]. The performance of APOLO is compared with that of the dropsonde and a ground-based radar profiler in Charlotte, North Carolina. Panoramic wind product displays for a selected date during the campaign are presented to show the trend of wind profile over an extensive observation period.

\section{DOPPLER WIND LIDAR SYSTEMS AT NASA LANGLEY}

The history of Doppler wind lidar systems and lidar signal processing at LaRC is quite extensive [2,8,12,17]. It includes the ground-based system Validation Lidar (VALIDAR) [9,10] and the airborne system DAWN [1,2]. VALIDAR is housed in a trailer for mobility and has been operational since 2002. It is also used for Differential Absorption Lidar (DIAL) measurements and analyses [12]. The next generation of VALIDAR is DAWN, which is a pulsed 2-micron coherent Doppler lidar system for airborne wind profiling. The first airborne demonstration of DAWN was in 2010 as part of the NASA's Genesis and Rapid Intensification Processes (GRIP) campaign based in Fort Lauderdale, Florida. The second flight demonstration was in November, 2012 based at LaRC, and the third one was during June 12 - July 23, 2013, also based at LaRC. The flight hours were approximately 130 hours on a DC-8 in 2010, 22 hours on a UC-12B in 2012, and 24 hours on a UC-12B in 2013.

Since VALIDAR is ground-based, it is moved to a location where wind measurements are to be made. The measurements are stationary in a fixed location with three LOSs typically - two elevated and orthogonal LOSs and one

\footnotetext{
${ }^{1}$ Jeffrey.Y.Beyon@nasa.gov; phone 1757 864-4249; fax 1757 864-7944; www.nasa.gov
} 
vertical. The measurement strategy with DAWN is similar to VALIDAR even though the number of LOSs with DAWN is allowed to vary. There is no INS/GPS data involved with VALIDAR for it is immobile during the data acquisition process. The wind profiling algorithm for VALIDAR is relatively simple using vector summation [10,13-16]. The lidar backscatter measurements with DAWN, however, are not as straightforward as VALIDAR because the DAWN system operates on a fast moving aircraft. Consequently, the INS/GPS data of the aircraft and the accurate orientation biases of the DAWN instrument play a critical role in not only data measurement but data analysis. Since the measurements are made on a moving platform, any slight misinterpretation of such information results in erroneous wind products [3,4].

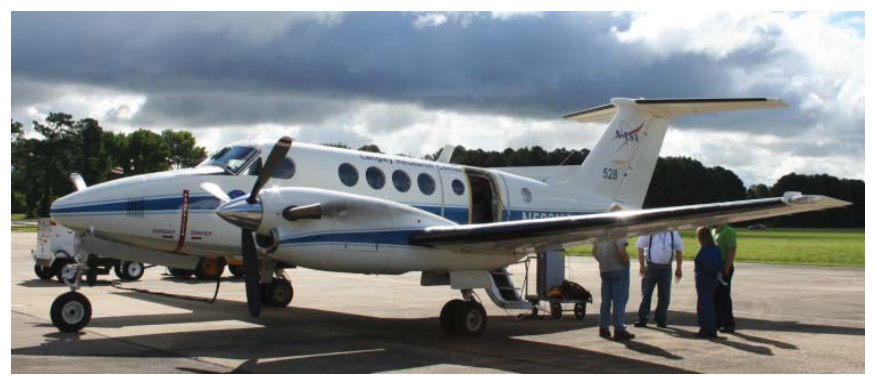

Figure 1 NASA's UC-12B. DAWN flew on this aircraft for the campaigns in 2012 and 2013 based at LaRC.

\section{AIRBORNE WIND PROFILING ALGORITHMS}

The wind profiling algorithm for VALIDAR is relatively simple and is based on vector summation. Since the data from VALIDAR are acquired at a fixed location, no information about motion or orientation of instrument is required in VALIDAR data analyses. The DAWN system, on the other hand, acquires data airborne on a fast moving platform, and the wind profiling algorithm for DAWN must take into consideration the movement and the orientation of the aircraft as well as the instrument in extracting wind parameters embedded in noisy lidar returns. An INS/GPS unit is used to obtain such information for DAWN. The INS/GPS unit is mounted to the DAWN instrument, and the offset between the INS/GPS reading and the actual orientation of the instrument must be compensated in estimating wind parameters in the airborne case.

In order to process the data from DAWN, there have been two versions of wind profiling algorithms developed at LaRC - APOLO and 5-directional method - and their high-level comparison can be found in [3]. Their sensitivity to noise, the framework of wind parameter extraction principles, and the number of LOSs used in each algorithm are quite distinctive. This paper focuses on the results from APOLO. APOLO utilizes the data from the INS/GPS unit attached to the DAWN instrument to compensate the adverse impact by the motion and orientation biases, and its performance has been validated by the comparison of APOLO wind products with those from the dropsonde and a radar profiler. APOLO uses the lidar returns reflected by a hard non-moving surface such as the ground to mitigate the impact of offsets.

\section{APOLO, DROPSONDE, AND RADAR}

The performance comparison of APOLO with dropsonde is done using the data from the GRIP campaign and with a radar profiler is done using the 2013 data while flying over a 915-MHz radar wind profiler near Charlotte, North Carolina [1]. In comparison of the performance, one must understand the fundamental differences in the wind products from an airborne wind lidar such as DAWN, and the dropsonde and a radar profiler. The wind profiling by DAWN is based on light backscatter and almost instantaneous while dropsondes use in-situ sensors and weather radars depend on radio waves to measure winds. The integration time of dropsondes and weather radars is far greater than wind lidars. The dropsondes also drift as they descend once deployed while making measurements. Therefore, we are using the wind products from the dropsonde and the radar profiler as one reference, not as the true golden wind measurements to validate the performance of APOLO.

Figure 2 shows the comparison of APOLO with dropsondes using the GRIP data. The offsets in role, pitch, yaw, 
azimuth, and elevation of DAWN instrument have been compensated using ground returns. One may expect a closer match at or near the aircraft altitude, and the graphs show an excellent match for all altitudes in general. Missing APOLO estimates around 4-9 km are due to the low signal-to-noise-ratio (SNR) caused by insufficient backscatter levels created by a combination of low atmospheric aerosol content and a faulty optical component.
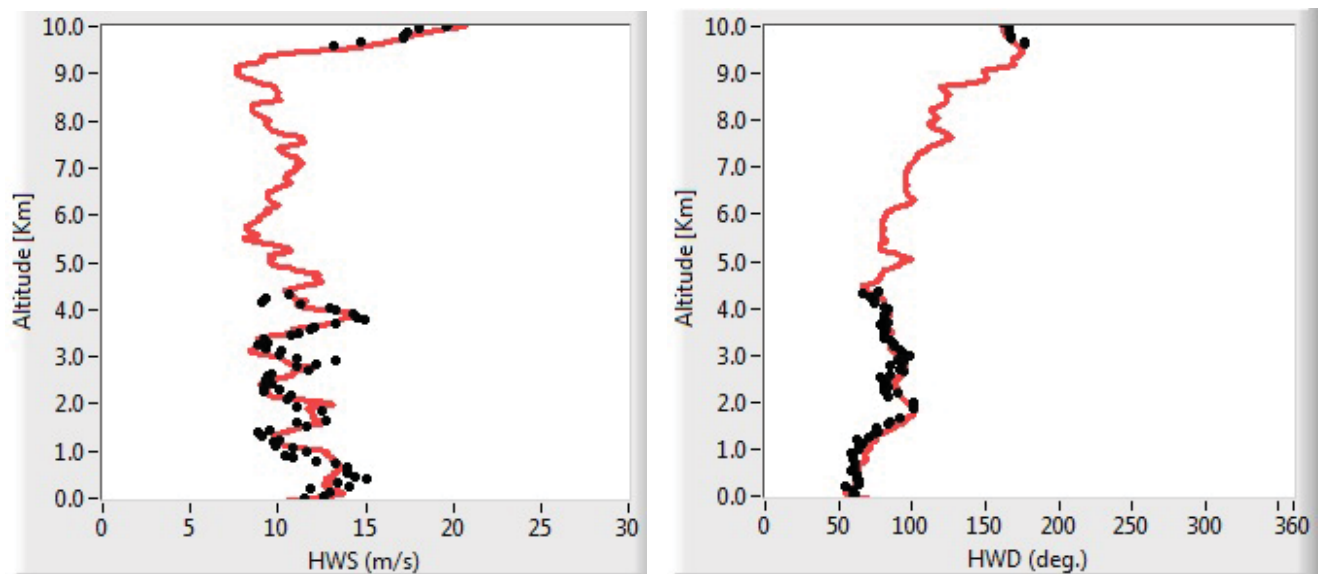

Figure 2 APOLO and Dropsonde. Offset compensation was applied. Data on September 1, 2010 during the GRIP mission on DC8. FFT size $=4096$ with zero padding. Altitude $=10,608$ meters. Solid line represents dropsonde data, and dots are the estimates from APOLO. HWS stands for horizontal wind speed and HWD, horizontal wind direction.

Figure 3 compares the APOLO wind parameters with those from the radar profiler. The UC-12B flew over the radar profiler in Charlotte, North Carolina while DAWN was making measurements. The integration time for DAWN was 30 seconds whereas 30 minutes for the radar profiler. The exact integration time period of the radar data is not known; however, a good match in wind direction is an indication of excellent performance of APOLO. Wind direction is extremely sensitive to the accuracy of wind measurements since it involves the trigonometric calculation of two wind measurements divided. Therefore, a good match in wind direction is more creditable than a good match in wind speed in evaluating the performance of APOLO. Figure 4 shows the flight path of the UC-12B on June 12, 2013 while DAWN was making measurements.
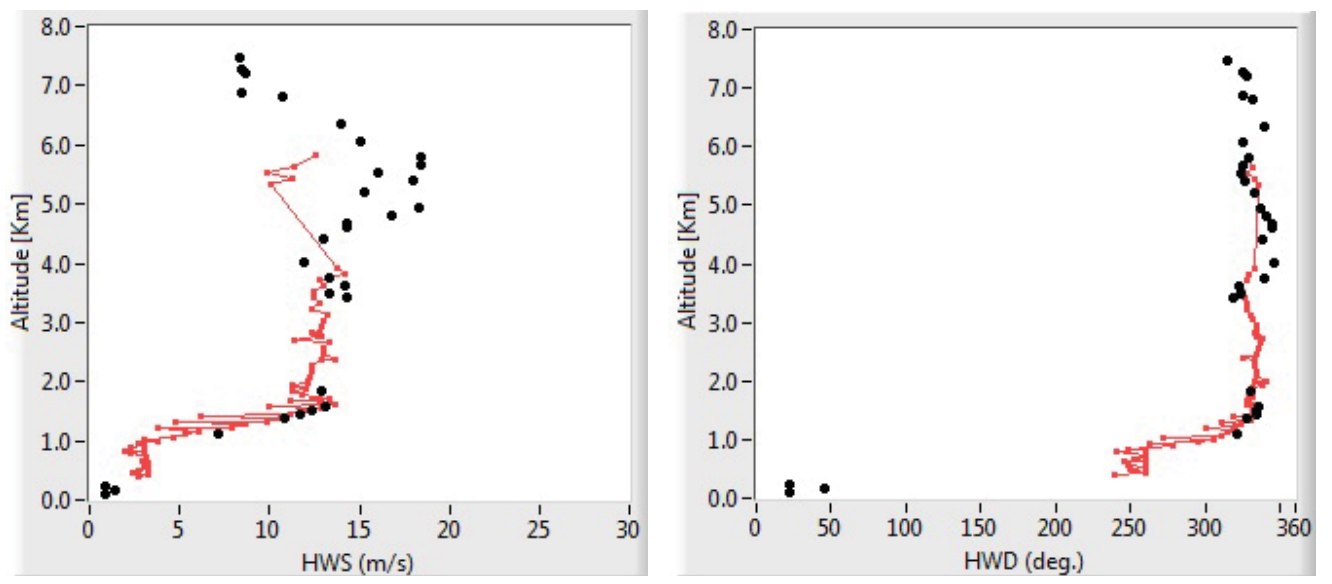

Figure 3 APOLO and ground-based radar profiler. Offset compensation was applied. Data on June 12, 2013 during a flight campaign on UC12B over Charlotte Radar. FFT size $=4096$ with zero padding. Altitude $=7,736$ meters. Solid line represents radar data, and dots are the estimates from APOLO. HWS stands for horizontal wind speed and HWD, horizontal wind direction.

The lidar is seen to make wind measurements from the aircraft altitude down to ground level. A cloud layer was encountered between 5 and 6-km altitude (which allowed radar measurements to be made in this altitude range). Despite the presence of the cloud, the lidar was able to retrieve enough signal under the clouds to make wind measurements. The 
clouds did degrade signal level significantly enough, though, to prevent lidar measurements in the 2 to $3.5-\mathrm{km}$ altitude range. There is good agreement where sensors have overlap and considering difference in integration time, which are 30 seconds for DAWN and 30 minutes for the radar.

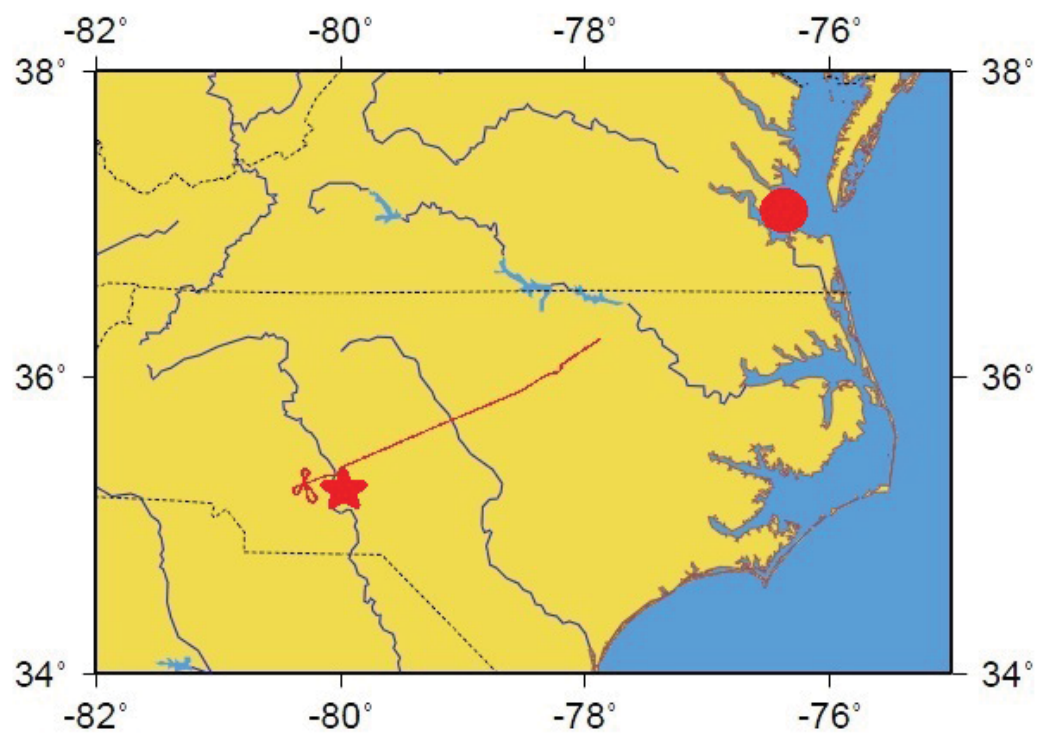

Figure 4 Flight path of UC12B on June 12, 2013. The map is shown by longitude and latitude, and the flight path is the red solid line. The star is the location of the radar in Charlotte, and the circle is NASA LaRC and Langley Air Force Base, where the aircraft took off.

Figure 5 shows the flight path of DAWN on June 26, 2013 - offshore of Maryland. This flight path was a test of a new technique for creating 3-dimensional maps of the wind vector over offshore areas being considered for wind farming. The aircraft is used to raster scan the wind energy area to sample the wind on many east-to-west transits.
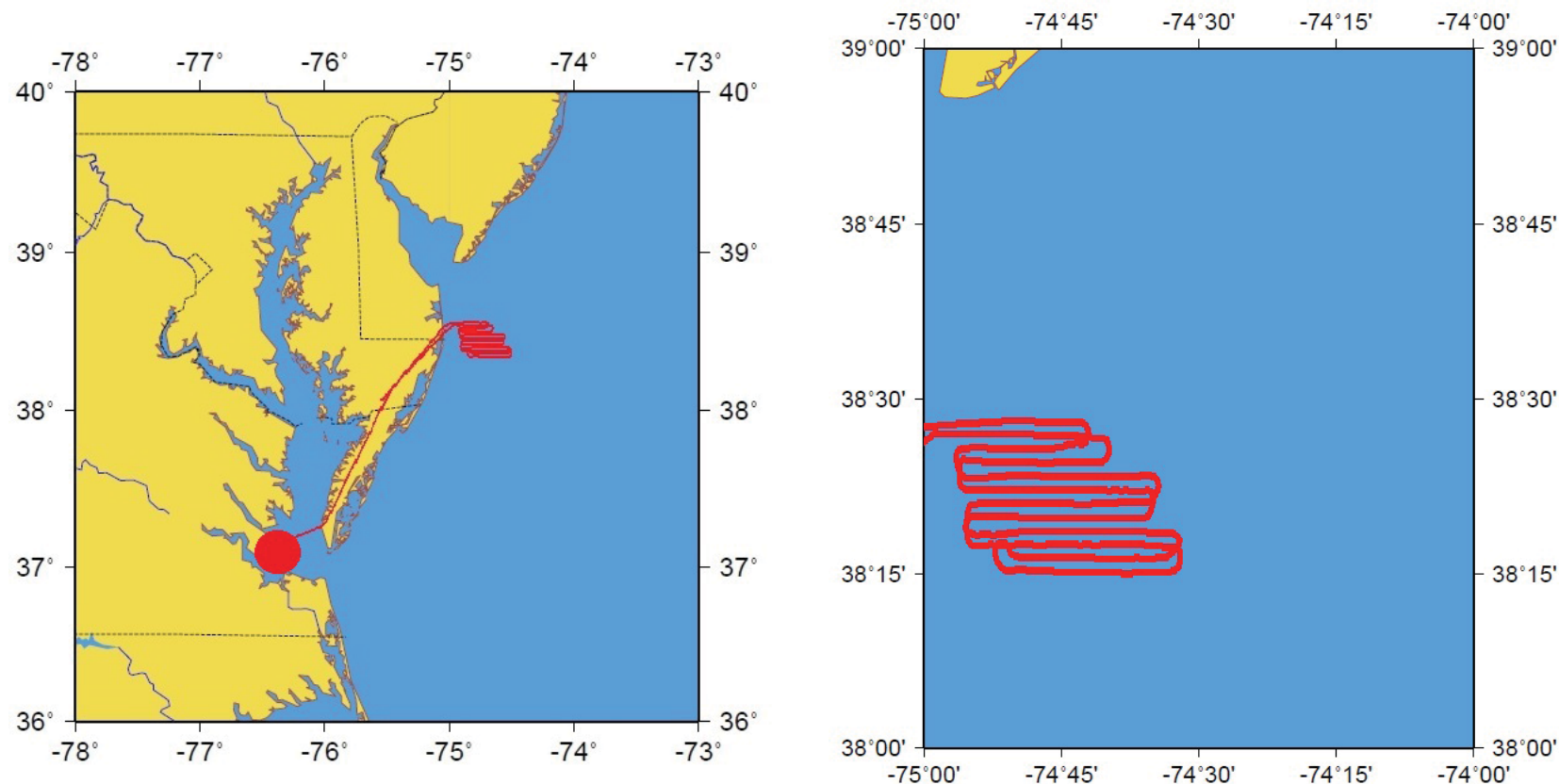

Figure 5 Flight path of UC12B on June 26, 2013. The map is shown by longitude and latitude, and the flight path is the red solid line. The circle is the location of NASA LaRC and Langley Air Force Base, where the aircraft took off. 
An example result of this new wind imaging technique is shown in Figure 6 of the wind vector at 100 -m above the ocean surface. The view at 100-m was chosen as this corresponds to the hub height of wind turbines that are being considered for installation in this area. A color coding algorithm shows contours of velocity, indicating geographic structure in the wind speed and direction. Such variations are likely to be relevant in considering development of an offshore wind farm.
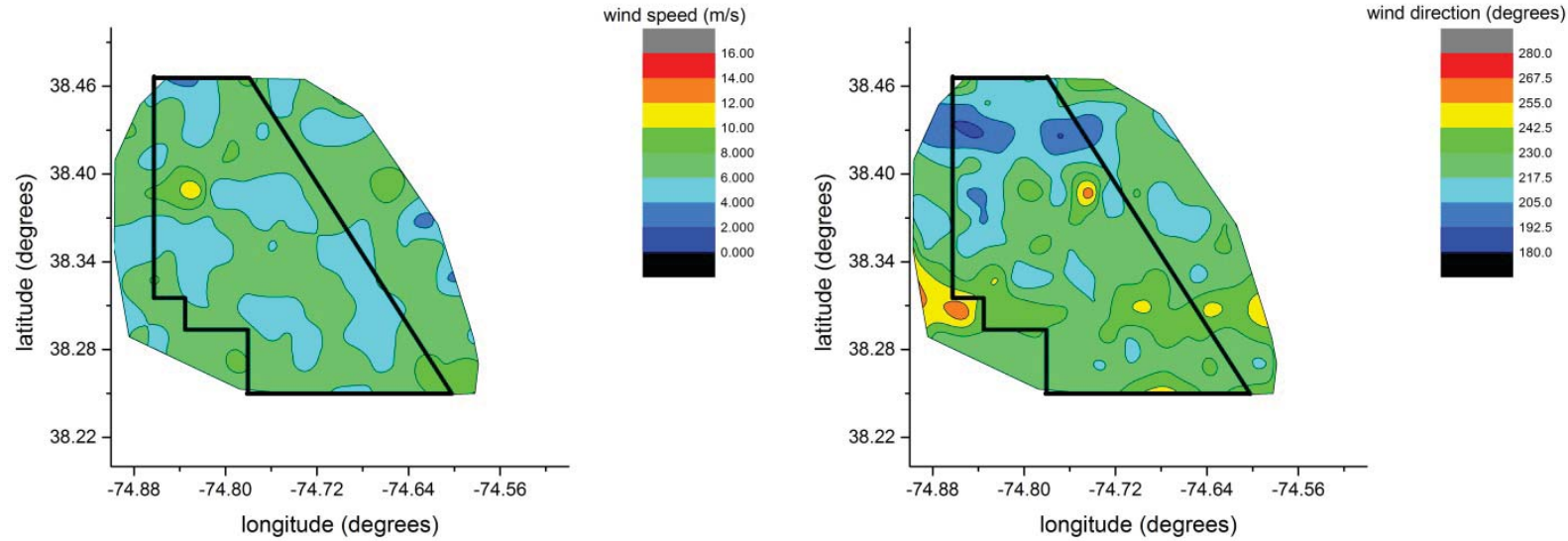

Figure 6 Map of wind vector at 100-m above ocean surface of the Maryland WEA measured on June 26, 2013

Figures 7-9 show panoramic displays of power, horizontal wind speed (HWS), and horizontal wind direction (HWD) for the DAWN data acquired on June 26, 2013. $x$-axis shows the Zulu time and $y$-axis indicates the altitude in $\mathrm{km}$. Each plot indicates values by color, which is shown to the right of each graph. In the flight experiment represented by Figures 7 through 9, lidar measurements began with the aircraft at 3-km altitude. Then the aircraft descended to 1.5-km altitude for a raster scan of the Maryland offshore wind energy area (from which the data of Figure 6 was derived). After overflying the Maryland offshore wind energy area (WEA), the aircraft ascended to 2.5-km altitude to return to Langley Air Force Base. Figure 7 shows the backscatter signal level from which areas of higher or lower aerosol density can be determined. Figure 8 indicates the wind speed at different altitudes. For example, over the ocean the wind speed close to the ocean surface is around $5-\mathrm{m} / \mathrm{s}$ up to around 500-m altitude. Above this layer, the wind speed increases to around $10-\mathrm{m} / \mathrm{s}$. Figure 9 indicates the wind direction, with variations seen in altitude of a similar pattern to that of wind speed. In all three panels of data of Figures 7 through 9, anomalous results appear as vertical lines. These errors occur when the aircraft is in sharp turns.
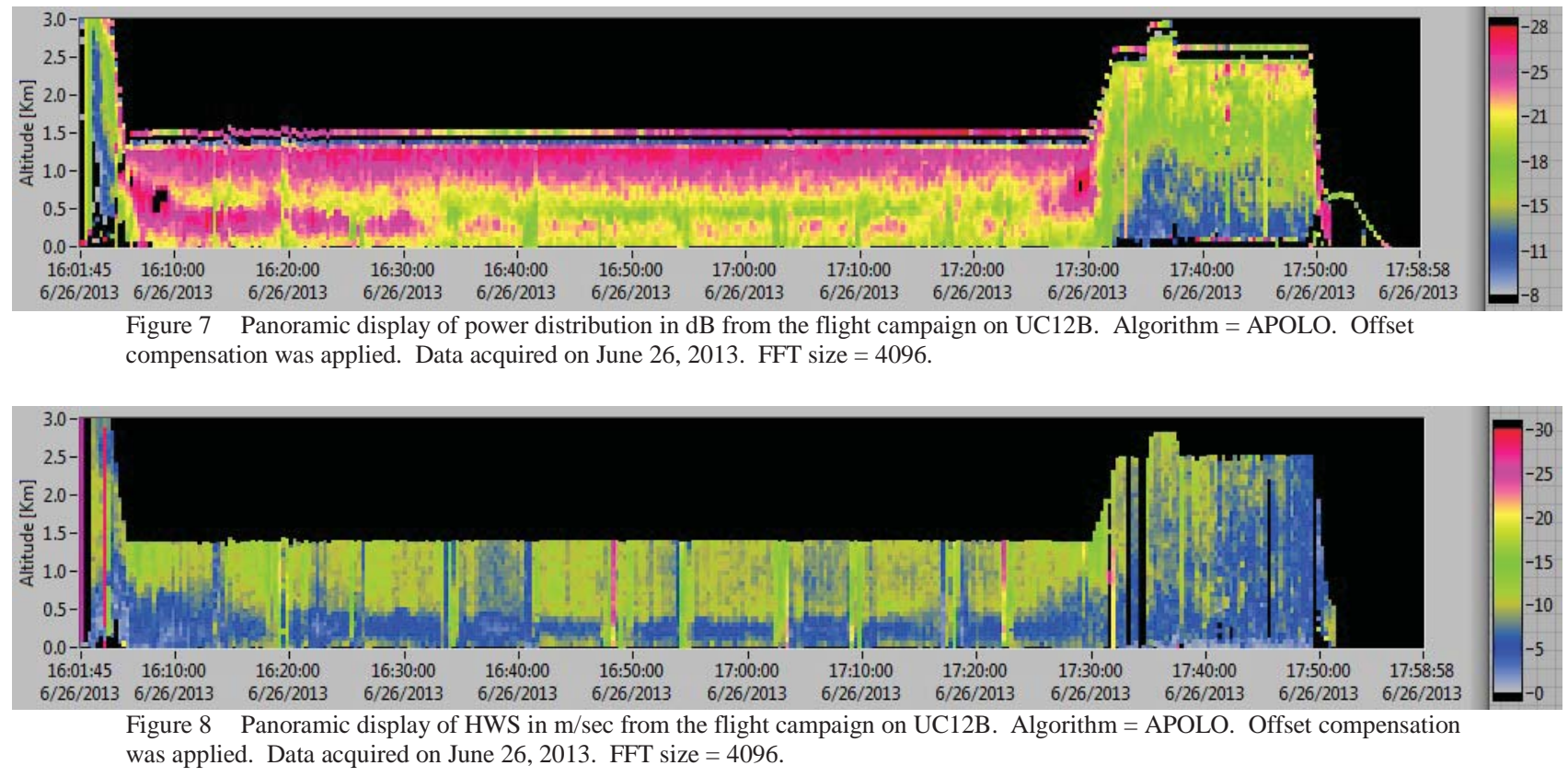


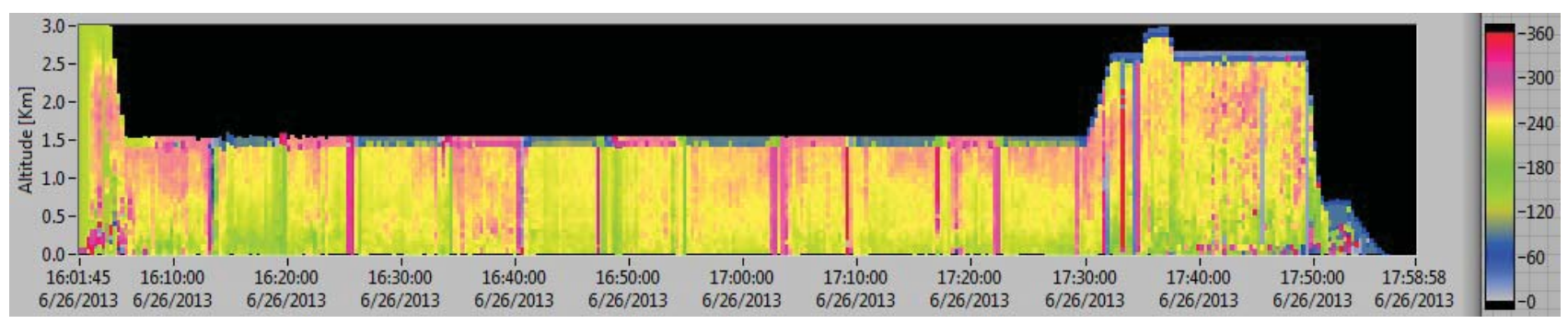

Figure 9 Panoramic display of HWD in degrees from the flight campaign on UC12B. Algorithm = APOLO. Offset compensation was applied. Data acquired on June 26, 2013. FFT size $=4096$.

\section{CONCLUSION}

The offshore wind measurements by the DAWN system are presented in this paper. The airborne wind profiling algorithm APOLO is applied and its wind parameters are presented using a panoramic display for the entire time span of observation. The performance of APOLO is illustrated by comparing its wind parameters with those from the dropsonde and the radar profiler in Charlotte, North Carolina. In such comparison, DAWN data from 2010 GRIP campaign and 2013 UC-12B flight demonstration are used. The comparison shows the excellent performance of APOLO in HWS and HWD estimates. APOLO utilizes the INS/GPS data in order to mitigate the adverse impact by aircraft motion and installation offsets. Using the wind products generated by APOLO, the wind energy areas (WEAs) are presented using the offshore wind measurements by DAWN. The flight paths of the DAWN system are shown to help understand the WEA analysis.

\section{ACKNOWLEDGMENT}

The authors are grateful for support from NASA Science Mission Directorate (SMD), the SMD Airborne Instrument Technology Transition program, the NASA SMD Earth Science Technology Office (ESTO) Instrument Incubator Program, the ESTO Laser Risk Reduction Program, Langley Research Center (LaRC), and the LaRC Engineering Directorate.

\section{BIBLIOGRAPHY}

[1] G. J. Koch, J. Y. Beyon, L. J. Cowen, M. J. Kavaya, and M. S. Grant, "3-Dimensional Wind Profiling of Offshore Wind Energy Areas with Airborne Doppler Lidar,” Journal of Applied Remote Sensing, Forthcoming 2014.

[2] M. J. Kavaya, J. Y. Beyon, G. J. Koch, M. Petros, P. J. Petzar, U. N. Singh, B. Trieu, and J. Yu, "The DAWN Airborne, Wind-Profiling, Coherent-Detection Lidar System: Overview, Flight Results, and Plans,” Journal of Atmospheric and Oceanic Technology (JTECH), Forthcoming 2014.

[3] J. Y. Beyon, G. J. Koch, M. J. Kavaya, and T. J. Ray, “Airborne Wind Profiling Algorithms for a Pulsed 2Micron Coherent Doppler Lidar at NASA Langley Research Center," in Proc. of the Defense and Security Symposium 2013 (8731-20), Baltimore, MD, April, 2013.

[4] J. Y. Beyon, G. J. Koch, and M. J. Kavaya, "Airborne Wind Profiling with the Data Acquisition and Processing System for a Pulsed 2-Micron Coherent Doppler Lidar System,” in Proc. of the Defense and Security Symposium 2012 (8379-22), Baltimore, MD, April, 2012.

[5] J. Y. Beyon, G. E. Arthur, G. J. Koch, and M. J. Kavaya, "Noise Whitening in Airborne Wind Profiling with a Pulsed 2-Micron Coherent Doppler Lidar at NASA Langley Research Center," in Proc. of the Defense and Security Symposium 2012 (8379-23), Baltimore, MD, April, 2012. 
[6] J. Y. Beyon, G. J. Koch, and M. J. Kavaya "Development of the Data Acquisition and Processing System for a Pulsed 2-Micron Coherent Doppler Lidar System,” in Proc. of the SPIE Asia-Pacific Remote Sensing (7860-9), Incheon, Republic of Korea, October, 2010.

[7] J. Y. Beyon, G. J. Koch, and M. J. Kavaya "Data Acquisition and Processing System for Airborne Wind Profiling with a Pulsed, 2-Micron, Coherent-Detection, Doppler Lidar System,” in Proc. of the Earth Science Technology Forum 2010, Arlington, VA, June, 2010.

[8] G.J. Koch, J.Y. Beyon, P.J. Petzar, M. Petros, J. Yu, B.C. Trieu, M.J. Kavaya, U.N. Singh, E.A. Modlin, B.W. Barnes, and B.B. Demoz, "Field Testing of a High-Energy 2-um Doppler Lidar,” Journal of Applied Remote Sensing 4, 043512, 2010.

[9] K. Vermeesch, G. Koch, B. Gentry, T. Bacha, H. Chen, and B. Demoz, "Comparisons of Ground-Based, Radiosonde, and Aircraft Wind Measurements at the Howard University Beltsville Research Site,” Wind Lidar Working Group Meeting, June, 2009.

[10] J. Y. Beyon, G. J. Koch, M. J. Kavaya, and M. Sahota, "Comparison of theoretical and empirical statistics of wind measurements with validation lidar (VALIDAR)," in Proc. of the Defense and Security Symposium 2008(6968-59), Orlando, FL, March, 2008.

[11] J. Y. Beyon and G. J. Koch, "Novel nonlinear adaptive Doppler-shift estimation technique for the coherent Doppler validation lidar,” Optical Engineering, Vol. 46, No. 1, pp. 016002-1 - 016002-10, January 2007.

[12] J. Y. Beyon, G. J. Koch, and S. Ismail "Signal processing techniques for heterodyne differential absorption lidar,” in Proc. of the Defense and Security Symposium 2007 (6567-53), Orlando, FL, April, 2007.

[13] G.J. Koch, J.Y. Beyon, B.W. Barnes, M. Petros, J. Yu, F. Amzajerdian, M.J. Kavaya, and U.N. Singh, "HighEnergy 2- $\mu m$ Doppler Lidar for Wind Measurements," Optical Engineering, Vol. 46(11), pp. 116201-1 116201-14, November, 2007.

[14] J. Y. Beyon and G. J. Koch, "Novel Nonlinear Adaptive Doppler Shift Estimation Technique (NADSET) for the Coherent Doppler Lidar System VALIDAR,” in Proc. of the Defense and Security Symposium 2006 (62361), Orlando, FL, April, 2006.

[15] J. Y. Beyon and G. J. Koch, "Resolution Study of Wind Parameter Estimates by a Coherent Doppler Lidar System,” in Proc. of the Defense and Security Symposium 2006 (6214-3), Orlando, FL, April, 2006.

[16] J. Y. Beyon and G. J. Koch, "Wind Profiling by a Coherent Doppler Lidar System VALIDAR with a Subspace Decomposition Approach,” in Proc. of the Defense and Security Symposium 2006 (6236-5), Orlando, FL, April, 2006.

[17] J. Y. Beyon, G. J. Koch, and Z. Li "Noise Normalization and Windowing Functions for VALIDAR in Wind Parameter Estimation,” in Proc. of the Defense and Security Symposium 2006 (6214-4), Orlando, FL, April, 2006.

[18] J. Yu, B.C. Trieu, E.A. Modlin, U.N. Singh, M.J. Kavaya, S. Chen, Y. Bai, P.J. Petzar, “1 J/pulse Q-switched 2 um solid-state laser,” Optics Letters 31, 462-462, 2006. 